\title{
Technical note: Rapid field test for the quantification of vitamin E, $\beta$-carotene, and vitamin A in whole blood and plasma of dairy cattle
}

\author{
Morteza H. Ghaffari, ${ }^{1}$ Katrin Bernhöft, ${ }^{2}$ Stephane Etheve, ${ }^{3}$ Irmgard Immig, ${ }^{3}$ Michael Hölker, ${ }^{1}$ \\ Helga Sauerwein, ${ }^{1}$ and Florian J. Schweigert ${ }^{4 *}$ \\ ${ }^{1}$ Institute of Animal Science, University of Bonn, 53115 Bonn, Germany \\ ${ }^{2}$ BioAnalyt $\mathrm{GmbH}, 14513$ Teltow, Germany \\ ${ }^{3}$ DSM Nutritional Products Ltd., 4303 Kaiseraugst, Switzerland \\ ${ }^{4}$ Department of Physiology and Pathophysiology, Institute of Nutritional Science, University of Potsdam, 14558 Nuthetal, Germany
}

\section{ABSTRACT}

Fast and easy tests for quantifying fat-soluble vitamins such as vitamin $\mathrm{E}$ and vitamin $\mathrm{A}$, as well as $\beta$-carotene, in whole blood without a need to preprocess blood samples could facilitate assessment of the vitamin status of dairy cattle. The objective of this study was to validate a field-portable fluorometer/ spectrophotometer assay for the rapid quantification of these vitamins in whole blood and plasma of dairy cows and calves. We measured the concentrations of vitamin $\mathrm{E}$ and $\beta$-carotene in whole blood and plasma from 28 dairy cows and 11 calves using the iCheck test (BioAnalyt GmbH, Teltow, Germany) and compared the results with the current analytical standard (HPLC) in 2 independent laboratories, one at the University of Potsdam (Germany) and at one at DSM Nutritional Products Ltd. (Kaiseraugst, Switzerland). For vitamin A, the HPLC measurements were done only in the laboratory in Germany. The whole-blood concentrations of vitamin $\mathrm{E}$ as determined by iCheck (blood-hematocritcorrected) ranged from 1.82 to $4.99 \mathrm{mg} / \mathrm{L}$ in dairy cows and 0.34 to $3.40 \mathrm{mg} / \mathrm{L}$ in calves. These findings were moderately correlated $\left(\mathrm{R}^{2}=0.66\right)$ with the values assessed by HPLC in dairy cattle (cows + calves). When calves were excluded, the correlation was higher $\left(\mathrm{R}^{2}=\right.$ 0.961). The $\beta$-carotene and vitamin $\mathrm{A}$ values obtained by the reference method HPLC were highly correlated with the iCheck methods in whole blood $\left(\mathrm{R}^{2}=0.99\right.$ and 0.88 , respectively). In plasma, we observed strong correlations between the concentrations assessed by iCheck and those of HPLC for vitamin $\mathrm{E}\left(\mathrm{R}^{2}=0.97\right)$,

Received April 5, 2019.

Accepted July 17, 2019.

*Corresponding author: florian.schweigert@uni-potsdam.de $\beta$-carotene $\left(R^{2}=0.98\right)$, and vitamin $A\left(R^{2}=0.92\right)$ in dairy cattle (cows + calves). For vitamin E, $\beta$-carotene, and vitamin $\mathrm{A}$, we compared the relationship between the differences obtained by the iCheck assay and the HPLC measurements, as well as the magnitude of measurements, using Bland-Altman plots to test for systematic bias. For all 3 vitamins, the differences values were not outside the $95 \%$ acceptability limits; we found no systematic error between the 2 methods for all 3 analytes.

Key words: vitamin, cow-side assay, HPLC, method comparison

\section{Technical Note}

Vitamin E, $\beta$-carotene, and vitamin A are important fat-soluble micronutrients required for animal growth and maintenance of the immune system in dairy cattle (Weiss et al., 1997; NRC, 2001). Both vitamin E and $\beta$-carotene play a major role in biological systems as antioxidants, preventing oxidative damage caused by reactive oxygen species (Traber and Atkinson, 2007). Low serum concentrations of vitamin $\mathrm{E}$ in early lactation are associated with increased risk of metabolic disorders in dairy cows (Weiss et al., 1997; Qu et al., 2013). Multiparous dairy cows with a left displacement of the abomasum were shown to have $40 \%$ lower circulating vitamin $\mathrm{E}$ concentrations in early lactation than their healthy counterparts (Mudron et al., 1997; Qu et al., 2013). In addition, dairy cows with low plasma concentrations of vitamin $\mathrm{E}(<3 \mu \mathrm{g} / \mathrm{mL})$ were reported to have a 9.4 times greater risk for clinical mastitis than cows with higher vitamin E concentrations (Weiss et al., 1997). In newborn calves, the plasma concentrations of vitamin $\mathrm{E}$ are extremely low relative to dairy cows (Herdt and Stowe, 1991).

Vitamin A, in the form of retinol, is associated with a specific transport protein-retinol-binding 
proteins - whose concentrations are tightly controlled and altered in case of deficiencies (Schweigert et al., 1990). $\beta$-Carotene is an important precursor of vitamin A (Schweigert, 1998) and is converted to retinol in the intestinal mucosa (Chew, 1987). Vitamin A and $\beta$-carotene are both involved in functions such as fertility (Kawashima et al., 2010), immune function, and health in general (Chew, 1993; Kume and Toharmat, 2001). Both vitamin $\mathrm{E}$ and $\beta$-carotene are transported in the blood associated with different lipoprotein fractions, and their circulating concentrations are closely associated with nutritional status (Schweigert et al., 1987).

Concentrations of vitamin $\mathrm{E}$, vitamin $\mathrm{A}$, and $\beta$-carotene decrease around calving (Goff et al., 2002; Calderón et al., 2007), partly due to their transfer into colostrum (Schweigert and Zucker, 1988; Goff et al., 2002; Weiss et al., 2009). The placental transfer of these components is limited, and thus tissue levels are low in newborn calves, making their intake with colostrum particularly important for a rapid fill-up of body stores (Hidiroglou 1989; Njeru et al., 1994). Low concentrations of vitamin $\mathrm{E}, \beta$-carotene, and vitamin A likely reflect the immune function and health of dairy cattle (Johansson et al., 2014). The status of fat-soluble vitamins and $\beta$-carotene should be monitored in dairy cattle and fast assessments of blood concentrations without the need for separating plasma would allow for rapid diagnostics and treatment. In this study, we describe a new assay for measuring vitamin $\mathrm{E}, \beta$-carotene, and vitamin $\mathrm{A}$ in the whole blood of dairy cattle (cows and calves) and compare the results with HPLC methods in plasma as a reference.

Whole blood was collected from the jugular veins of German Holstein cows $(\mathrm{n}=28)$ and calves $(\mathrm{n}=11)$ as a cross-section of dairy cattle from the Frankenforst research station of the Faculty of Agriculture, University of Bonn (Königswinter, Germany). Blood collection was done in the framework of the ethical approval obtained from the local authority (No. 84-02.04.2015. A139), following European Union legislation, and in accordance with the German Animal Welfare Act (Federal Republic of Germany, 2014). As a reference method for the iCheck fluorometer/spectrophotometric assay (BioAnalyt GmbH, Teltow, Germany), we performed HPLC-based analyses in 2 independent laboratories: at the University of Potsdam (Germany; Lab DE) and at DSM Nutritional Products Ltd. (Kaiseraugst, Switzerland; Lab CH). Blood samples were collected into tubes containing EDTA. Samples were shipped on ice, but not frozen, to Potsdam. The vitamin analyses in whole blood and the separation of plasma were performed within $2 \mathrm{~h}$ after the arrival of the samples.
Aliquots of the plasma were frozen and shipped to Lab $\mathrm{CH}$.

In Lab DE, the HPLC analyses were conducted using a modified gradient reverse-phase system (Waters, Eschborn, Germany) as previously described (Schweigert et al., 2003). Vitamin E, $\beta$-carotene, and vitamin A were extracted from the aqueous suspension with $n$-hexane, dried, dissolved in solvent solution, and separated on a reverse-phase column (ReproSil 70 C18 column, $200 \times 3.0 \mathrm{~mm}$; particle size $5 \mu \mathrm{m}$; Dr. Maisch GmbH, Ammerbuch, Germany). The solvent system consisted of solvent A with methanol, and solvent B with ethyl acetate, at a flow rate of $0.5 \mathrm{~mL} / \mathrm{min}$ (LC 20-AD pump; Shimadzu, Kyoto, Japan) and a column temperature of $40^{\circ} \mathrm{C}$. The gradient was 0 to $3 \mathrm{~min}, 0 \%$ solvent B; $3.1 \mathrm{~min}, 50 \%$ solvent B; $10 \mathrm{~min}, 50 \%$ solvent B; 10.1 min, $0 \%$ solvent B, and 15 min stop. Vitamin E (as $\alpha$-tocopherol) and $\beta$-carotene were identified based on their retention times in comparison to an external standard (Sigma-Aldrich, Munich, Germany) using a photodiode array detector (PDA SPDM-20A; Shimadzu). Calibration was done with traceable standard analyte materials. Vitamin E, $\beta$-carotene, and vitamin A were quantified by measuring the absorption at 290 $\mathrm{nm}, 450 \mathrm{~nm}$, and $325 \mathrm{~nm}$, respectively.

In Lab $\mathrm{CH}$, plasma proteins were precipitated with ethanol. Vitamin $\mathrm{E}$ and $\beta$-carotene were then extracted from the aqueous suspension with $n$-hexane and butylhydroxytoluene. After centrifugation, an aliquot of the organic phase was dried, re-dissolved in solvent solution and injected onto a reversed-phase (Ascentis Express C18, Supelco $150 \mathrm{~mm} \times 3.0 \mathrm{~mm}, 2.1-\mu \mathrm{m}$ particle size) UHPLC system (1290 Infinity; Agilent, Santa Clara, CA). Detection was performed with a UV/Vis detector (1290 Infinity DAD; Agilent) at $450 \mathrm{~nm}$ for $\beta$-carotene and with a fluorescence detector (1260 Infinity FLD Spectra; Agilent) at excitation wavelength of $298 \mathrm{~nm} /$ emission wavelength of $328 \mathrm{~nm}$ for vitamin E. The mobile phase was a solution of acetonitrile: tetrahydrofurane:methanol:1\% ammonium acetate solution (684:220:68:28, vol/vol/vol/vol) with ascorbic acid. The system ran under isocratic mode at a flow rate of $0.5 \mathrm{~mL} / \mathrm{min}$ for $16 \mathrm{~min}$. The retention times for vitamin $\mathrm{E}$ and $\beta$-carotene were $4.0 \mathrm{~min}$ and $8.5 \mathrm{~min}$, respectively.

Data acquisition, identification, integration, and calculation were conducted using Chromeleon 7.2 software (Thermo Scientific, Waltham, MA). Quantification was performed by applying external calibration.

The correlations between each of the 2 HPLC methods are shown separately in Supplemental Figure S1 (https://doi.org/10.3168/jds.2019-16755) and were very strong $\left(R^{2}=0.96\right.$ for vitamin $E$ and $R^{2}=0.98$ for 
$\beta$-carotene; both $P<0.001)$ in cows' plasma and also in calves' plasma $\left(\mathrm{R}^{2}=0.91\right.$ for vitamin $\mathrm{E}$ and $\mathrm{R}^{2} 0.82$ for $=\beta$-carotene; $P<0.001)$. Vitamin $\mathrm{A}$ was assessed in Lab DE only.

We determined vitamin E, $\beta$-carotene, and vitamin A using the iCheck fluorometer/spectrophotometer in whole blood and in plasma samples of the whole blood. The analytical process is based on an extraction unit that contains all of the necessary chemicals for extraction and separation, as well as a portable measuring device based on fluorometric and spectroscopic properties (iCheck Vitamin E/A/Carotene; BioAnalyt $\mathrm{GmbH}$ ). We performed the analysis of vitamin $\mathrm{E}$, $\beta$-carotene, and vitamin $\mathrm{A}$ in whole blood samples and plasma using these innovative assay systems as follows. Whole blood and plasma samples were each mixed for $1 \mathrm{~min}$, and $500 \mu \mathrm{L}$ of the sample was injected into the reagent solution vial using the provided applicator. Then, the reagent vials were shaken vigorously for 10 $\mathrm{s}$ and allowed to stand without refrigeration for $5 \mathrm{~min}$ until complete phase separation. Finally, vitamin E, $\beta$-carotene, and vitamin A were extracted into the organic solvent and measured using the respective iCheck assay device. In the whole blood sample mode, results were adjusted to plasma levels based on an average hematocrit level of $32 \%$, as has been shown to be valid for cattle (Raila et al., 2012). Based on the analytical principle that includes no chromatographic separation step, the spectrophotometric and fluorometric determination provides the sum of all tocopherols, all carotenoids, and the sum of retinol and retinyl esters, which are quantitatively summarized as retinol equivalents (Raila et al., 2012; Elom et al., 2015).

Data (plasma sample) were analyzed using the PROC MIXED procedure of SAS (version 9.4; SAS Institute Inc., Cary, NC). Before analysis, all data were tested for normality of distribution by evaluating Shapiro-Wilk statistics using the UNIVARIATE procedure of SAS. The $\beta$-carotene and vitamin $\mathrm{A}$ data from dairy calves were $\log 10$-transformed. The model consisted of treatment (iCheck vs. HPLC) as a fixed effect and animal (cow or calves) as a random effect. We determined the associations between HPLC and the results of the rapid test system using the Pearson correlation coefficient in SigmaPlot (version 12.5; Systat Software Inc., San Jose, CA). We created Bland-Altman plots in Excel (Microsoft, Redmond, WA) to graphically determine the analytical accuracy of the 2 analytical methods; the plots include a solid horizontal line showing the mean bias between the 2 methods (HPLC and iCheck), as well as the $95 \%$ confidence interval of the agreement. Statistical significance was declared at $P \leq 0.05$.

The whole blood concentrations as determined by iCheck in dairy cows ranged from 1.82 to $4.99 \mathrm{mg} / \mathrm{L}$ for vitamin $\mathrm{E}, 2.09$ to $8.15 \mathrm{mg} / \mathrm{L}$ for $\beta$-carotene, and 184 to $336 \mu \mathrm{g} / \mathrm{L}$ for vitamin $\mathrm{A}$. In calves, the range was 0.34 to $3.40 \mathrm{mg} / \mathrm{L}$ for vitamin E, 0.05 to 2.52 $\mathrm{mg} / \mathrm{L}$ for $\beta$-carotene, and 2.09 to $8.15 \mathrm{mg} / \mathrm{L}$ for vitamin A. We found no differences between the 2 methods (iCheck fluorometer vs. HPLC) for the average concentrations of plasma vitamin E, $\beta$-carotene, and vitamin A in dairy cows and calves (Table 1), supporting the good agreement between the 2 methods. In whole blood, a moderate correlation for vitamin $\mathrm{E}$ $\left(\mathrm{R}^{2}=0.66, P=0.05\right.$, average from Lab DE and Lab $\mathrm{CH}$, Figure 1a) and strong correlations for $\beta$-carotene $\left(\mathrm{R}^{2}=0.99, P<0.001\right.$, average from Lab DE and Lab $\mathrm{CH}$, Figure $1 \mathrm{~b})$ and vitamin $\mathrm{A}\left(\mathrm{R}^{2}=0.88, P<0.001\right.$, Lab CH, Figure 1c) were indicated between iCheck (blood-hematocrit-corrected) and HPLC methods in dairy cattle (cows + calves). The lower association in the case of vitamin $\mathrm{E}$ was attributable to the calf data (Supplemental Table S1; https://doi.org/10 .3168/jds.2019-16755). When limiting calculation of

Table 1. Plasma concentrations of vitamin E, $\beta$-carotene, and vitamin A assessed by iCheck fluorometer (BioAnalyt GmbH, Teltow, Germany) and HPLC in dairy cows $(\mathrm{n}=28)$ and calves $(\mathrm{n}=11)$

\begin{tabular}{lcccc}
\hline & \multicolumn{2}{c}{ Treatment } & & \\
\cline { 2 - 3 } Item & iCheck & HPLC & SEM & P-value \\
\hline Vitamin E $(\mathrm{mg} / \mathrm{L})$ & 3.00 & 3.42 & 0.14 & 0.13 \\
$\quad$ Cows & 1.59 & 1.68 & 0.13 & 0.76 \\
$\quad$ Calves & & & & \\
$\beta$-Carotene $(\mathrm{mg} / \mathrm{L})$ & 4.79 & 5.08 & 0.23 & 0.53 \\
$\quad$ Cows & 0.38 & 0.36 & 0.13 & 0.65 \\
$\quad$ Calves & & & & \\
Vitamin A $\left(\mu \mathrm{g}\right.$ of $\left.\mathrm{RE}^{1} / \mathrm{L}\right)$ & 298.9 & 279.6 & 7.21 & 0.18 \\
$\quad$ Cows & 138.4 & 111.9 & 12.8 & 0.31 \\
Calves & & & \\
\hline
\end{tabular}

${ }^{1}$ Retinol equivalent (RE) considers the vitamin A activity of different forms of vitamin A (1 $\mu \mathrm{g}$ of retinol or retinyl palmitate equals $1 \mu \mathrm{g}$ or $0.55 \mu \mathrm{g}$ of $\mathrm{RE}$, respectively). 


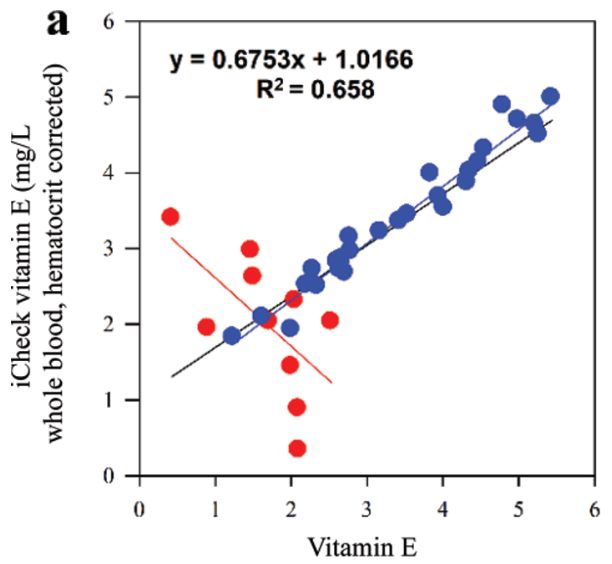

(HPLC, average from Lab DE and $\mathrm{Lab} \mathrm{CH}, \mathrm{mg} / \mathrm{L}$ )

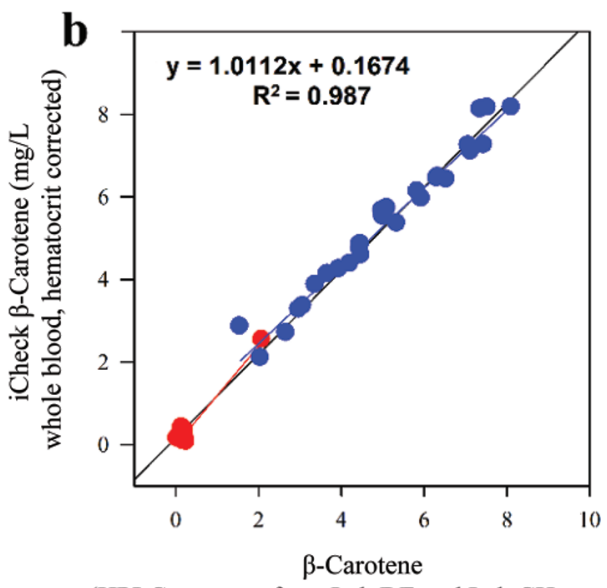

(HPLC, average from Lab DE and $\mathrm{Lab} \mathrm{CH}, \mathrm{mg} / \mathrm{L}$ )

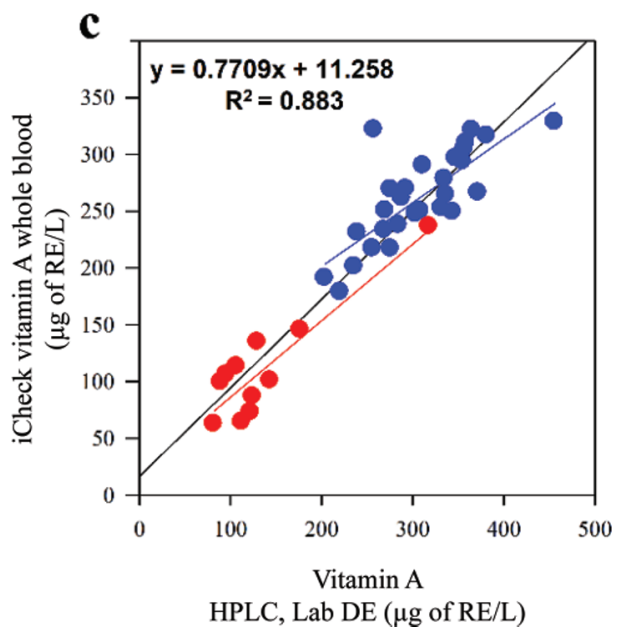

Figure 1. Correlation between concentrations of (a) vitamin $\mathrm{E}$, (b) $\beta$-carotene, and (c) vitamin A assessed by iCheck (BioAnalyt GmbH, Teltow, Germany) in whole blood vs. HPLC in whole blood. Red and blue dots designate samples from dairy calves and dairy cows, respectively; the respective regression lines follow the same color code. Regression across all samples is shown as a black line. Lab $\mathrm{DE}=$ University of Potsdam, Germany; Lab CH = DSM Nutritional Products Ltd., Kaiseraugst, Switzerland. Retinol equivalent (RE) considers the vitamin A activity of different forms of vitamin A $(1 \mu \mathrm{g}$ of retinol or retinyl palmitate equals $1 \mu \mathrm{g}$ or $0.55 \mu \mathrm{g}$ of $\mathrm{RE}$, respectively). the correlation to the data of cows only, the coefficient of correlation was comparable $\left(\mathrm{R}^{2}=0.961\right)$ and also close to results from previous studies (Raila et al., 2012, 2017). The measurement of vitamin E, vitamin $\mathrm{A}$, and $\beta$-carotene by HPLC in blood plasma requires the separation of blood cells from plasma. Using the new method, this centrifugation step is not necessary, so whole blood can be readily used to measure these components. However, as discussed below, the precision of the iCheck vitamin E measurement at the low range of concentrations in calves seems to be compromised in whole blood.

When comparing the concentrations assessed in plasma from all animals (cows and calves) via iCheck and HPLC (average from Lab DE and Lab CH, Figure $2 \mathrm{a}$ and $\mathrm{b}$ ), we observed strong correlations for vitamin $\mathrm{E}\left(\mathrm{R}^{2}=0.97, P<0.001\right), \beta$-carotene $\left(\mathrm{R}^{2}=0.98, P<\right.$ 0.001 ), and vitamin A (HPLC analyses from Lab CH only; $\mathrm{R}^{2}=0.92, P<0.001$, Figure $\left.2 \mathrm{c}\right)$. When separating cow and calf data, the $\mathrm{R}^{2}$ values ranged from 0.77 (vitamin $\mathrm{A}$ in cow samples) to 0.93 ( $\beta$-carotene in cow samples). A detailed compilation of these results is provided in Supplemental Figures S2 to S4 and Supplemental Table S1 (https://doi.org/10.3168/ jds.2019-16755). The strong correlations between the iCheck fluorometer and the HPLC method when using plasma exclusively confirm the applicability of the new method. The iCheck fluorometer can thus be used when centrifugation is possible but HPLC methods are not available. However, preparing plasma from whole blood on a farm is often not possible, and using whole blood overcomes this limitation. The general applicability of the iCheck fluorometer for whole blood was demonstrated in the present study by the high correlations $\left(\mathrm{R}^{2}\right.$ $>0.9$ ) with the HPLC reference methods for vitamin $A$ and $\beta$-carotene. Similar correlations were shown for vitamin $\mathrm{E}$, but only when limiting the comparison to samples from cows. In calves, vitamin E concentrations are lower than in cows, and the precision of iCheck might be compromised at that low range when using whole blood. However, given the high correlations observed when comparing calves' plasma vitamin E values from iCheck versus the HPLC data $\left(\mathrm{R}^{2}=0.87\right)$, the low concentration range alone does not explain the lower correlation in whole blood. Moreover, the hematocrit values were not different between cows and calves, so a bias due to the constant hematocrit setting in the iCheck can be ruled out. We can only speculate that the whole blood matrix in calves differs from that of cows, possibly related to the different stages of development (preweaning status with ruminant status not fully developed compared with mature ruminants). It cannot be excluded that the blood matrix of calves might interfere to some extent with the iCheck technol- 


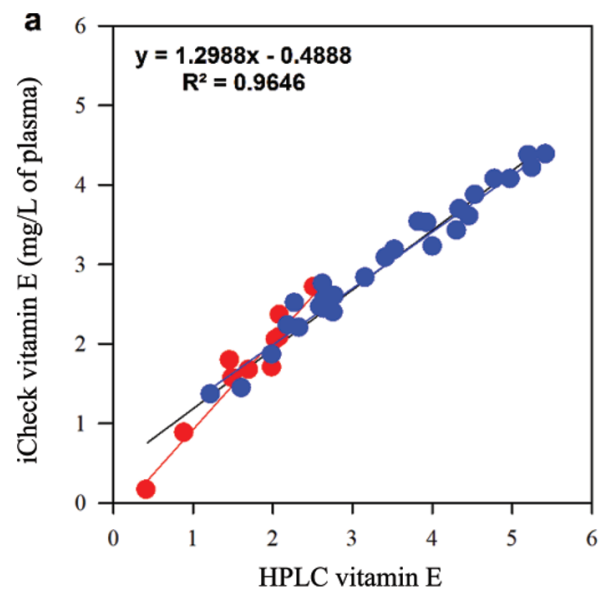

(average $\mathrm{Lab} \mathrm{DE}$ and $\mathrm{Lab} \mathrm{CH}, \mathrm{mg} / \mathrm{L}$ of plasma)

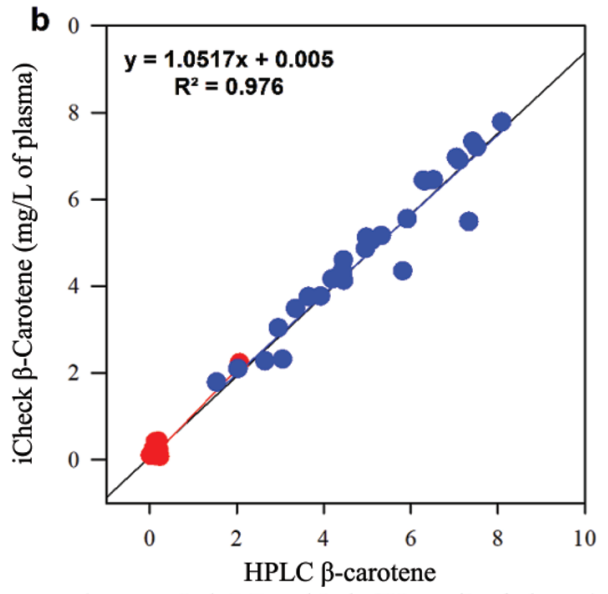

(average $\mathrm{Lab} \mathrm{DE}$ and $\mathrm{Lab} \mathrm{CH}, \mathrm{mg} / \mathrm{L}$ of plasma)

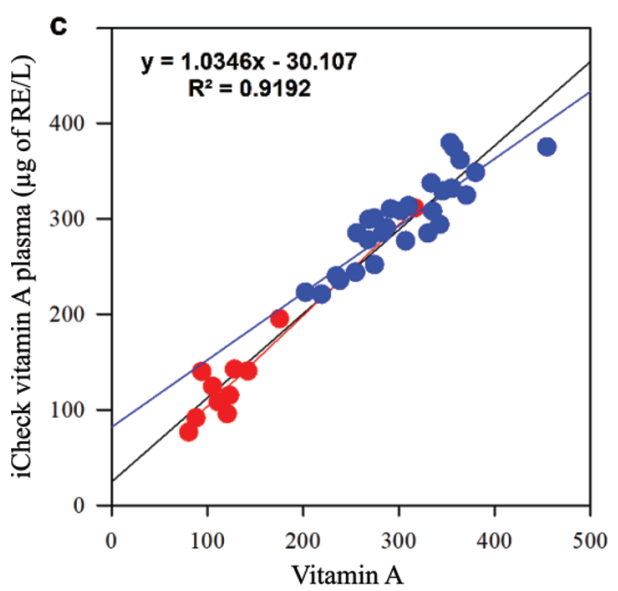

HPLC Lab DE ( $\mu$ g of RE/L)

Figure 2. Correlation between concentrations of (a) vitamin E, (b) $\beta$-carotene, and (c) vitamin A assessed by iCheck (BioAnalyt $\mathrm{GmbH}$, Teltow, Germany) in plasma vs. HPLC in plasma. Red and blue dots designate samples from dairy calves and dairy cows, respectively; the respective regression lines follow the same color code. Regression across all samples is shown as a black line. Lab DE = University of Potsdam, Germany; Lab CH = DSM Nutritional Products Ltd., Kaiseraugst, Switzerland. Retinol equivalent (RE) considers the vitamin A activity of different forms of vitamin A (1 $\mu \mathrm{g}$ of retinol or retinyl palmitate equals $1 \mu \mathrm{g}$ or $0.55 \mu \mathrm{g}$ of $\mathrm{RE}$, respectively).

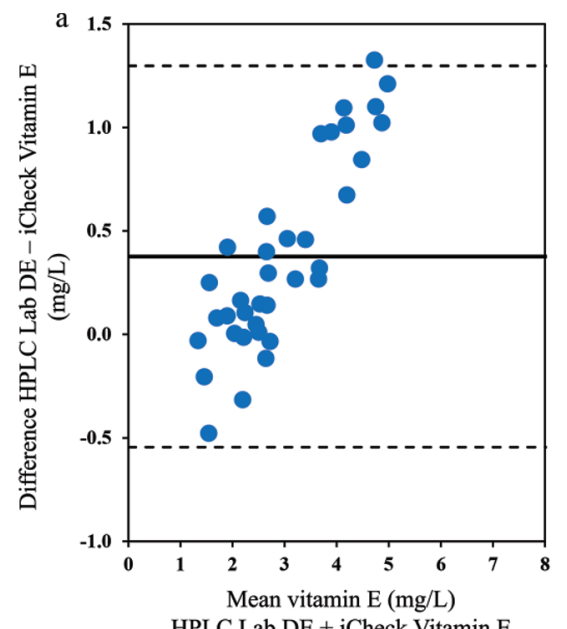

HPLC Lab DE + iCheck Vitamin E

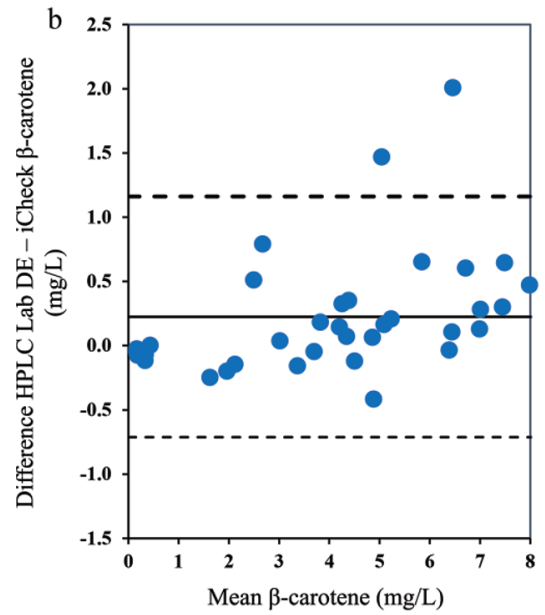

HPLC Lab DE + iCheck $\beta$-carotene

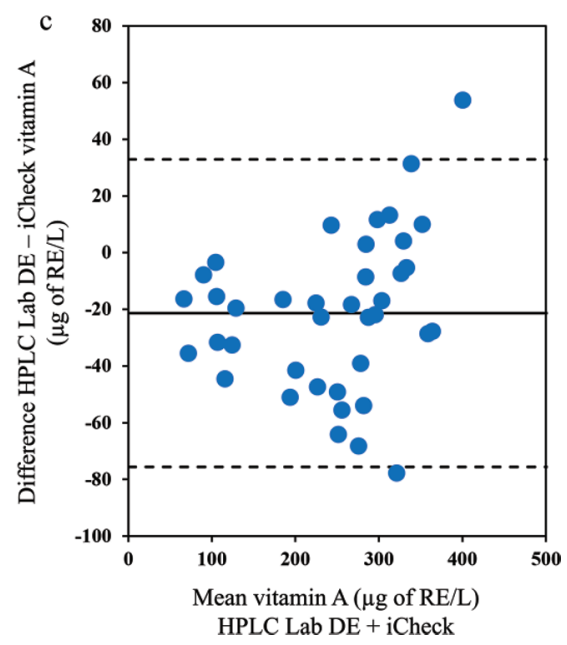

Figure 3. Bland-Altman plots showing the mean difference (solid line) and the $95 \%$ confidence interval (dashed lines) for the (a) vitamin $\mathrm{E}$, (b) $\beta$-carotene, and (c) vitamin A values in plasma obtained by HPLC and iCheck (BioAnalyt GmbH, Teltow, Germany) in dairy cattle $($ cow + calf $)$. Lab DE $=$ University of Potsdam, Germany; Lab CH = DSM Nutritional Products Ltd., Kaiseraugst, Switzerland. Retinol equivalent (RE) considers the vitamin A activity of different forms of vitamin $\mathrm{A}(1 \mu \mathrm{g}$ of retinol or retinyl palmitate equals $1 \mu \mathrm{g}$ or $0.55 \mu \mathrm{g}$ of $\mathrm{RE}$, respectively). 
ogy. Nevertheless, even though the correlation of whole blood iCheck versus HPLC values was less good in calves than in cows, this correlation was still significant at a moderate level.

The Bland-Altman plots showed that the differences in vitamin $\mathrm{E}$ (Figure $3 \mathrm{a}), \beta$-carotene (Figure $3 \mathrm{~b}$ ), and vitamin A (Figure 3c) values in Lab DE were not outside the $95 \%$ acceptability limits, suggesting no significant systematic error between the 2 methods (iCheck fluorometer vs. HPLC). Based on the Bland-Altman plots, no systematic error and a good level of agreement occurred for vitamin E (Supplemental Figure S2; (https://doi.org/10.3168/jds.2019-16755), $\beta$-carotene (Supplemental Figure S3), and vitamin A (Supplemental Figure S4) values in Lab DE between the 2 methods (iCheck fluorometer vs. HPLC) and only $5 \%$ of the differences in measured values fell outside the $95 \%$ acceptability limits for $\beta$-carotene in dairy cows.

\section{CONCLUSIONS}

In this study, all fat-soluble analytes were measured in whole blood and plasma from dairy cows and calves. Comparison of the iCheck fluorometer with the HPLC method as a reference method for measuring vitamin $\mathrm{E}$, vitamin $\mathrm{A}$, and $\beta$-carotene in the whole blood and plasma of dairy cattle showed a very good agreement, precision, and high accuracy. With the exception of vitamin E measurements in calves, whole blood samples can be directly used and the test can be performed in less than 5 minutes very easily, even at cow-side, using a field-portable fluorometer/spectrophotometer (iCheck) without further sample preparation. The ease of the test and the handling of the vials and the precalibrated measuring device also enables people with less training to perform a highly accurate test with results that comparable to HPLC, the gold standard. Based on this new development, the concentrations of vitamin $\mathrm{E}, \beta$-carotene, and vitamin $\mathrm{A}$ in blood can be used as nutritional biomarkers to directly optimize nutritional interventions at the farm, together with stakeholders such as veterinarians, farmers, nutritional advisors, and feed consultants.

\section{ACKNOWLEDGMENTS}

Technical support in analyzing the sample by Andrea Hurtinne (University of Potsdam, Germany) and Anja Weber (BioAnalyt GmbH, Teltow, Germany) is gratefully acknowledged. FJS is a shareholder of BioAnalyt $\mathrm{GmbH}$.

\section{REFERENCES}

Calderón, F., B. Chauveau-Duriot, P. Pradel, B. Martin, B. Graulet, M. Doreau, and P. Nozière. 2007. Variations in carotenoids, vitamins $\mathrm{A}$ and $\mathrm{E}$, and color in cow's plasma and milk following a shift from hay diet to diets containing increasing levels of carotenoids and vitamin E. J. Dairy Sci. 90:5651-5664.

Chew, B. P. 1987. Symposium: Immune function: Relationship of nutrition and disease control. Vitamin $\mathrm{A}$ and $\beta$-carotene on host defense. J. Dairy Sci. 70:2732-2743.

Chew, B. P. 1993. Role of carotenoids in the immune response. J. Dairy Sci. 76:2804-2811.

Elom, A.K., el M. Imane, B. Kaoutar, el K. Khalid, el H. Asmaa, A. Mehdi, el H. Noureddine, and A. Hassan. 2015. Comparison of a fluorometric assay kit with high-performance liquid chromatography for the assessment of serum retinol concentration. Afr. Health Sci. 15:641-646. https://doi.org/10.4314/ahs.v15i2.43.

Federal Republic of Germany. 2014. Tierschutzgesetz. Accessed Aug. 14, 2019. https://www.gesetze-im-internet.de/tierschg/ BJNR012770972.html.

Goff, J. P., K. Kimura, and R. L. Horst. 2002. Effect of mastectomy on milk fever, energy, and vitamins $\mathrm{A}, \mathrm{E}$, and $\beta$-carotene at parturition. J. Dairy Sci. 85:1427-1436.

Herdt, T. H., and H. D. Stowe. 1991. Fat-soluble vitamin nutrition for dairy cattle. Vet. Clin. North Am. Food Anim. Pract. 7:391-415.

Hidiroglou, M. 1989. Mammary transfer of vitamin E in dairy cows. J. Dairy Sci. 72:1067-1071.

Johansson, B., K. Persson Waller, S. K. Jensen, H. Lindqvist, and E. Nadeau. 2014. Status of vitamins E and A and $\beta$-carotene and health in organic dairy cows fed a diet without synthetic vitamins. J. Dairy Sci. 97:1682-1692. https://doi.org/10.3168/jds.2013-7388.

Kawashima, C., S. Nagashima, K. Sawada, F. J. Schweigert, A. Miyamoto, and K. Kida. 2010. Effect of beta-carotene supply during close-up dry period on the onset of first postpartum luteal activity in dairy cows. Reprod. Domest. Anim. 45:e282-e287.

Kume, S., and T. Toharmat. 2001. Effect of colostral $\beta$-carotene and vitamin A on vitamin and health status of newborn calves. Livest. Prod. Sci. 68:61-65.

Mudron, P., J. Rehage, H. P. Sallmann, M. Mertens, H. Scholz, and G. Kovac. 1997. Plasma and liver alpha-tocopherol in dairy cows with left abomasal displacement and fatty liver. Zentralbl. Veterinarmed. A 44:91-97.

National Research Council. 2001. Nutrient Requirements of Dairy Cattle. 7th rev. ed. Natl. Acad. Sci. Washington, DC.

Njeru, C. A., L. R. Mc, N. S. Dowell, S. B. Wilkinson, L. S. Linda, L. S. Rojas, and S. N. Williams. 1994. Pre- and postpartum supplemental DL-a-tocopheryl acetate effects on placental and mammary vitamin E transfer in sheep. J. Anim. Sci. 72:739-745.

Qu, Y., K. Lytle, M. G. Traber, and G. Bobe. 2013. Depleted serum vitamin E concentrations precede left displaced abomasum in early-lactation dairy cows. J. Dairy Sci. 96:3012-3022. https://doi .org/10.3168/jds.2012-6357.

Raila, J., F. Enjalbert, R. Mothes, A. Hurtienne, and F. J. Schweigert. 2012. Validation of a new point-of-care assay for determination of $\beta$-carotene concentration in bovine whole blood and plasma. Vet. Clin. Pathol. 41:119-122.

Raila, J., C. Kawashima, H. Sauerwein, N. Hülsmann, C. Knorr, A. Miyamoto, and F. J. Schweigert. 2017. Validation of blood vitamin A concentrations in cattle: Comparison of a new cow-side test (iCheck ${ }^{\mathrm{TM}}$ FLUORO) with high-performance liquid chromatography (HPLC). BMC Vet. Res. 13:126.

Schweigert, F. J. 1998. Metabolism of carotenoids in mammals. Pages 249-284 in Carotenoids Vol. 3: Biosynthesis and Metabolism. G. Britton, S. Liaaen-Jensen, and H. Pfander, ed. Birkhäuser Verlag, Basel, Switzerland.

Schweigert, F. J., W. A. Rambeck, and H. Zucker. 1987. Transport of $\beta$-carotene by the serum lipoproteins in cattle. J. Anim. Physiol. Anim. Nutr. (Berl.) 57:162-167.

Schweigert, F. J., O. A. Ryder, W. A. Rambeck, and H. Zucker. 1990. The majority of vitamin A is transported as retinyl esters in the 
blood of most carnivores. Comp. Biochem. Physiol. A Comp. Physiol. 95:573-578.

Schweigert, F. J., B. Steinhagen, J. Raila, A. Siemann, D. Peet, and U. Buscher. 2003. Concentrations of carotenoids, retinol, and alpha-tocopherol in plasma and follicular fluid of women undergoing IVF. Hum. Reprod. 18:1259-1264.

Schweigert, F. J., and H. Zucker. 1988. Transfer of beta-carotene into colostrum in the cow. Int. J. Vitam. Nutr. Res. 58:246-247.

Traber, M. G., and J. Atkinson. 2007. Vitamin E, antioxidant and nothing more. Free Radic. Biol. Med. 43:4-15. https://doi.org/10 .1016/j.freeradbiomed.2007.03.024.
Weiss, W. P., J. S. Hogan, D. A. Todhunter, and K. L. Smith. 1997. Effect of vitamin E supplementation in diets with a low concentration of selenium on mammary gland health of dairy cows. J. Dairy Sci. 80:1728-1737.

Weiss, W. P., J. S. Hogan, and D. J. Wyatt. 2009. Relative bioavailability of all-rac and RRR vitamin $\mathrm{E}$ based on neutrophil function and total $\alpha$-tocopherol and isomer concentrations in periparturient dairy cows and their calves. J. Dairy Sci. 92:720-731. 